\title{
Temporal patterning of neural synchrony in the basal ganglia in Parkinson's disease
}

Shivakeshavan Ratnadurai-Giridharan ${ }^{1}$, S. Elizabeth Zauber $^{2}$, Robert M. Worth ${ }^{1,3}$, Thomas Witt $^{3}$, Sungwoo Ahn ${ }^{1,5}$, Leonid L. Rubchinsky ${ }^{1,4}$ *

${ }^{1}$ Department of Mathematical Sciences and Center for Mathematical Biosciences, Indiana UniversityPurdue University Indianapolis, Indianapolis, IN, USA

${ }^{2}$ Department of Neurology, Indiana University School of Medicine, Indianapolis, IN, USA

${ }^{3}$ Department of Neurosurgery, Indiana University School of Medicine, Indianapolis, IN, USA

${ }^{4}$ Stark Neurosciences Research Institute, Indiana University School of Medicine, Indianapolis, IN, USA

${ }^{5}$ Present address: Department of Mathematics, East Carolina University, Greenville, NC, USA

\section{* Corresponding author:}

Leonid L. Rubchinsky, PhD

Department of Mathematical Sciences, Indiana University Purdue University Indianapolis Stark Neurosciences Research Institute, Indiana University School of Medicine

402 N. Blackford St, Indianapolis, IN 46202, USA

Tel.: +1-317 274-9745

Fax: +1-317-274-3460

E-mail: leo@math.iupui.edu

This is the author's manuscript of the article published in final edited form as:

Ratnadurai-Giridharan, S., Zauber, S. E., Worth, R. M., Witt, T., Ahn, S., \& Rubchinsky, L. L. (2016). Temporal patterning of neural synchrony in the basal ganglia in Parkinson's disease. Clinical Neurophysiology, 127(2), 1743-1745. http://doi.org/10.1016/j.clinph.2015.09.005 
Parkinson's disease (PD) is associated with elevated beta-band synchrony and oscillations in cortical and subcortical circuits including subthalamic nucleus (STN) and internal pallidum (GPi) (Hammond et al., 2007). The origin and nature of parkinsonian beta-band synchrony are poorly understood. We previously described temporal patterning of beta-band synchrony in STN (Park et al., 2010). Here, we explore and compare neural synchronization in GPi vs. STN. These nuclei attract special attention, being targets for surgical intervention. Moreover, STN may have a special role in the generation and expression of pathological synchrony because of its mutual excitatory-inhibitory connections with external pallidum. Although STN and GPi differ in function (excitatory vs. inhibitory), anatomical architecture, and connectivity, they both exhibit parkinsonian beta-band synchrony. Comparative analysis of synchrony properties may help in understanding the involvement of both nuclei in pathological parkinsonian neural activity.

This study includes eight PD patients undergoing microelectrode-guided DBS electrodes implantation. The study was approved by Indiana University IRB; patients provided informed consent. Four patients had GPi DBS (all male, age $56 \pm 7$ years, disease duration $11 \pm 3$ years,

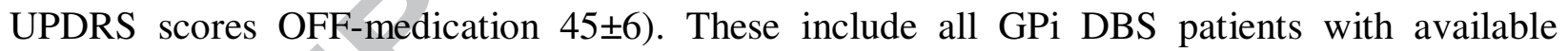
appropriate data. Four other patients had STN DBS (one female, age $69 \pm 6$ years, disease duration $7 \pm 3$ years, UPDRS scores OFF-medication 53 \pm 9 ). Electrophysiological recordings were performed with Guideline 4000 (FHC), modified to record spiking units and LFP. The average duration of recorded episodes was $225 \pm 45$ s. The procedures were described earlier (Park et al., 2010). 
The time-series analysis followed (Park et al., 2010). After extracting single units, spiking and LFP signals were band-pass filtered to the beta-band (defined here as 10-30 Hz). To detect oscillatory episodes, a signal-to-noise ratio criterion (Park et al., 2010) was used. The phases were reconstructed using Hilbert transform (Hurtado et al., 2004). Fig. 1(A, B) shows examples of the data. Fig. 1(C) shows the sines of the phases $\phi_{\text {spikes }}(t)$ and $\phi_{L F P}(t)$. The spiking/LFP synchrony strength was computed using a phase-locking index:

$$
\gamma=\left\|\frac{1}{N} \sum_{j=1}^{N} e^{i \varphi_{j}}\right\|^{2}
$$

where $\varphi_{j}=\phi_{\text {spikes }}\left(t_{j}\right)-\phi_{L F P}\left(t_{j}\right)$. We considered this index for spiking/LFP synchronization for both GPi and STN. The average (for the sample of all recordings in all patients) value of the synchrony index for STN was found to be 0.16 while for GPi, it was 0.14 . An ANOVA test indicated no significant difference between LFP-Spike synchronization strength in STN and GPi.

Temporal patterning of synchronization was characterized by the distribution of desynchronization durations (Park et al., 2010; Ahn et al., 2011). Briefly, whenever the phase of one signal (spiking unit) crosses an arbitrary threshold the value of the phase of the other signal (LFP) is recorded. We utilize episodes with significant phase-locking ( $\mathrm{p}<0.05$, estimated with surrogates (Hurtado et al., 2004; Park et al., 2010). Since some level of phase synchronization is present, there is a preferred value of the phase difference. Dynamics is considered to be desynchronized, when the actual phase difference deviates from the preferred phase difference by more than $\pi / 2$ as in (Park et al., 2010; Ahn et al., 2011). In this approach the duration of the desynchronized episodes is measured in cycles of the oscillations. This approach distinguishes between a large number of short desynchronizations, a small number of long desynchronizations 
and the spectrum of possibilities in between even if they all yield the same average value of synchronization strength.

Fig. 1(D) presents desynchronization durations distributions. Distributions have mode=1 and decay sharply. The desynchronizations lasting for one cycle of oscillations (synch/out of synch/synch) outnumber the next duration, two cycles, by more than a factor of two. The large number of desynchronizations may bring overall synchrony to low levels, but this low level is reached via many short desynchronizations (as opposed to a few long desynchronized episodes). Cycle 1 duration probability is significantly larger than all other probabilities (one-way ANOVA, $\mathrm{p}<10^{-5}$ ) for both GPi and STN. Thus our analysis indicates that the neural synchronization in GPi as well as in STN is punctuated by numerous episodes of desynchronized activity, most of which are very short-lasting.

In general, a given synchronization strength may be reached with many short or few long desynchronizations, or many possibilities in between (Park et al., 2010; Ahn et al. 2011). However, in the parkinsonian basal ganglia, the moderate synchrony is achieved due to frequent and short interruptions of the synchronized activity, which easily destabilizes and easily reestablishes itself exhibiting intermittent dynamics.

A similar patterning of STN synchrony was reported earlier (Park et al., 2010). So we performed comparative analysis of STN and GPi synchrony. Distributions of desynchronization durations in STN and GPi (Fig. 1D) are not significantly different (multivariate ANOVA, p=0.8). Thus synchrony in GPi and STN exhibits essentially identical temporal patterning. As a check, we 
performed similar analysis for alpha and theta frequency bands. Desynchronization duration distributions are statistically different $(\mathrm{p}<0.05)$ in between alpha and beta and between theta and beta bands in both STN and GPi activity. In particular, the average desynchronization duration for the beta-band is 1.94 cycles while the average desynchronization duration for alpha and theta bands are lower at 1.43 cycles and 1.29 cycles respectively. The decay of the distributions (how quickly the chances to observe a desynchronized event decrease with its duration) is frequencyspecific too. This indicates that the beta-band dynamics is different from other bands, which fits with the special role of beta in parkinsonism.

Note that the average ages of STN and GPi groups are different and this difference may have an unknown effect on the results. However we suppose that age difference is more likely to increase the difference between STN and GPi, yet out results indicate their similarity. We also note that we have only 4 patients per group, which may negatively affect statistical significance. However, STN group includes 26 different brain locations, from where good data were recorded, and GPi group has 20 different locations. Furthermore, the total number of desynchronization events is 1660 for STN group and 1335 for GPi group. Hence, the results regarding the properties of desynchronizations are obtained from a small sample of patients but a large sample of desynchronizations.

STN and GPi are very different anatomically: GPi is an inhibitory structure with intranuclear connectivity and inhibitory striatal input, STN is an excitatory structure (likely without intranuclear connectivity). LFPs (believed to be largely formed by synaptic currents) are thus likely to arise in STN and GPi in different ways. Yet, the similarity of temporal patterning of 
synchrony points to deep similarities in the organization and structure of underlying dynamics (Ahn et al., 2011). Thus the lack of statistically significant differences in the distribution of desynchronization events (and in the average level of synchrony strength) suggests that both STN and GPi belong to a functionally connected group of circuits supporting pathological betaband synchronized oscillations in PD. Together with the observations of cortico-subcortical interactions (Marreiros et al., 2013) our results support the view that pathological synchronous beta-band oscillations are a non-local phenomena supported by functionally connected internuclear networks of the brain. 


\section{Conflicts of Interest}

None of the authors have potential conflicts of interest to be disclosed.

\section{Acknowledgements}

The study was supported by IU-CRG and the Indiana University Health - Indiana University School of Medicine Strategic Research Initiative. 


\section{References}

Ahn S, Park C, Rubchinsky LL. Detecting the temporal structure of intermittent phase locking. Phys Rev E Stat Nonlin Soft Matter Phys 2011; 84:016201.

Hammond C, Bergmann H, Brown P. Pathological synchronization in Parkinson's disease: networks, models and treatments. Trends Neurosci 2007; 30:357-364.

Hurtado JM, Rubchinsky LL, Sigvardt KA. Statistical method for detection of phase-locking episodes in neural oscillations. J Neurophysiol 2004; 91:1883-1898.

Marreiros AC, Cagnan H, Moran RJ, Friston KJ, Brown P. Basal ganglia-cortical interactions in Parkinsonian patients. Neuroimage 2013; 66:301-310.

Park C, Worth RM, Rubchinsky LL. Fine temporal structure of beta oscillations synchronization in subthalamic nucleus in Parkinson's disease. J Neurophysiol 2010; 103:2707-2716. 


\section{Figure Legend}

Figure 1. (A) and (B) present examples of the spiking and filtered spiking signal and raw and filtered LFP signal respectively. The filtered signals are shown as solid black lines. (C) presents the sines of the phases of these filtered signals (dotted line - spiking, solid line - LFP). (D) presents the histogram of probabilities of desynchronization durations (with standard errors) for STN (grey) and GPi (white). Desynchronization durations are measured in cycles of oscillations. For the duration $>8$, all durations are pooled together so that the last bin accounts for all long durations longer than 8 cycles. 
(A)

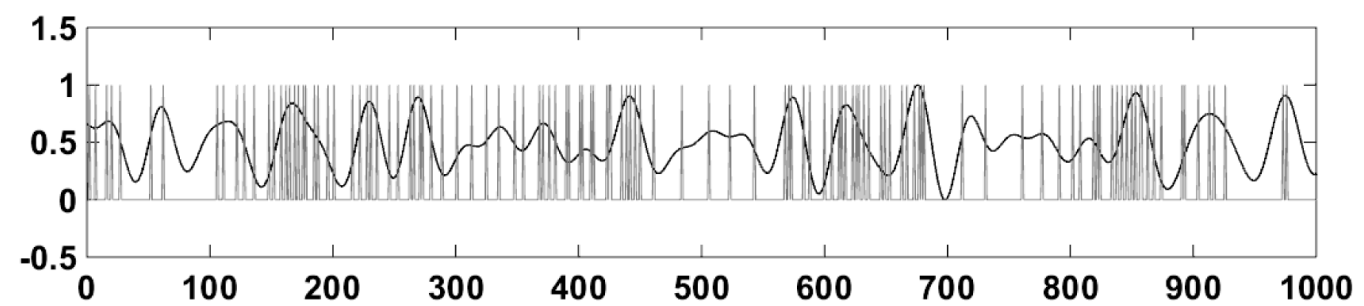

(B)

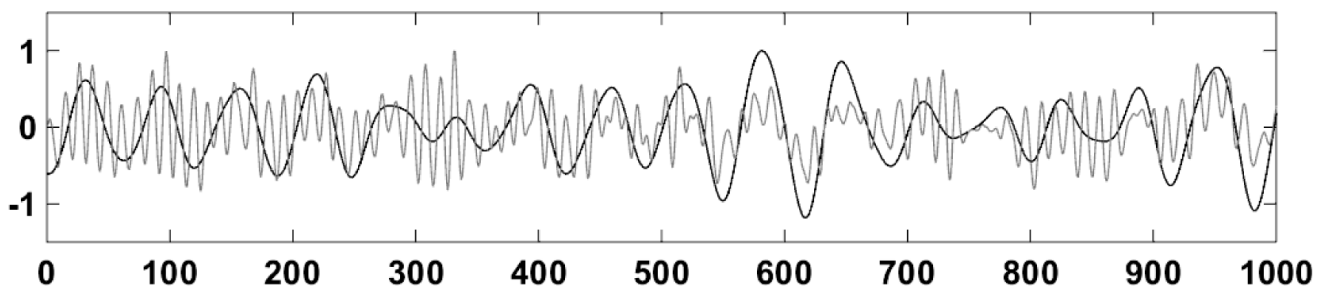

(C)

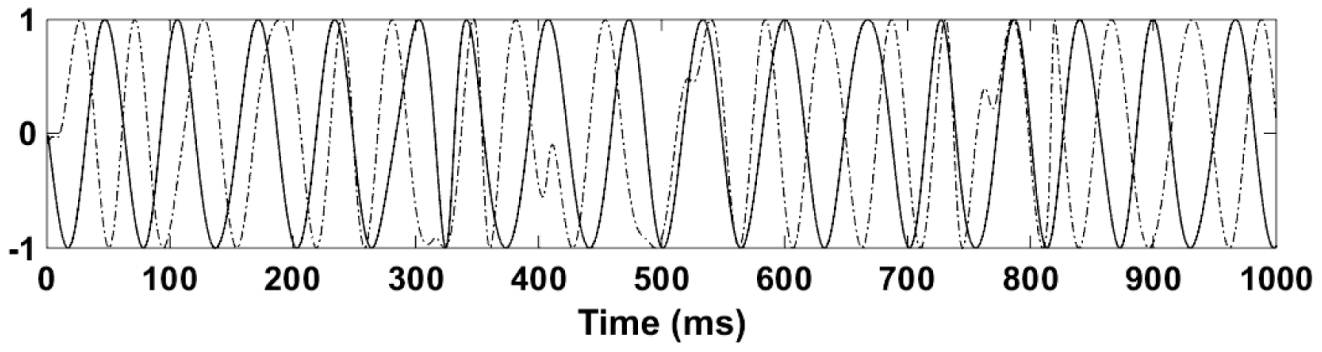

(D)

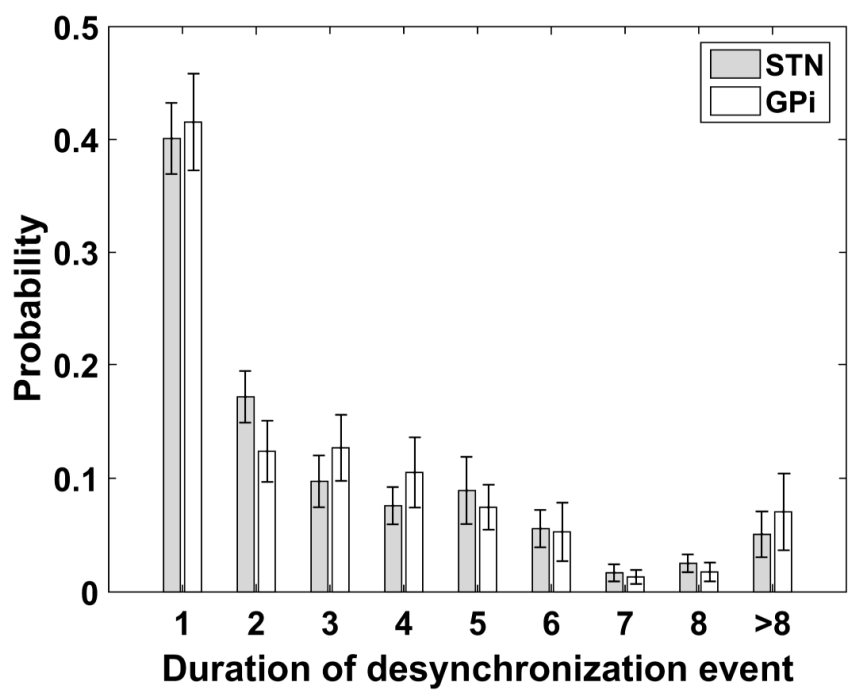

